\title{
The Role of Thyroid Hormones in the Central Nervous System
}

\author{
Maryam Borhani Haghighi ${ }^{1,2}$, Hoda Pasand Mojdeh ${ }^{3}$, Fatemeh Alipour ${ }^{\text {* }}$ \\ ${ }^{1}$ Shefa Neuroscience Research Center, Khatam Alanbia Hospital, Tehran, Iran \\ ${ }^{2}$ Department of Anatomy, School of Medicine, Tehran University of Medical Sciences, Tehran, Iran \\ ${ }^{3}$ School of Biology, College of Science, University of Tehran, Tehran, Iran
}

\section{ABSTRACT}

Introduction: The thyroid hormones have an important effect on the development and maturation of the mammalian central nervous system before and after birth. Thyroid hormones, triiodothyronine and thyroxine, adjust the processes of brain differentiation, such as synaptogenesis, dendritic and axonal growth, migration of neuronal cells and myelination. Any impairment in thyroid function and changes in the supply of thyroid hormones during development of nervous system leads to severe and irreversible alterations in the structure and function of the brain. Timing and duration of thyroid hormone deficiency affect development of different parts of the central nervous system. However, Neurological symptoms are commonly observed in adulthood with both hyperthyroidism and hypothyroidism. Conclusion: Understanding the mechanisms of the effect of thyroid hormones on embryo and adult neurogenesis is essential for improving the treatment of neurological disorders.

*Corresponding Author: Fatemeh Alipour

E-mail: Fatemehalipoor11@gmail.com 
نقش هورمونهاى تيروئيد در سيستم عصبى مركزى

مريم برهانى حقيقى "'، هدى بسند مزده"، فاطمه على يور "

'مركز تحقيقات علوم اعصاب شفا، بيمارستان خاتمالانبياء، تهران، ايران

rَّروه آناتومى، دانشكده يزشكى، دانشحاه علوم يزشكى تهران، تهران، ايران

"دانشكده زيست شناسى، يرديس علوم، دانشكاه تهران، تهران، ايران

اطلاعات مقاله:

تاريخ بذيرش: || شهريور عهس|

اصلاحيه: זr خرداد عوسات ماله:

تاريخ دريافت: FF اسفند هوس|

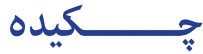

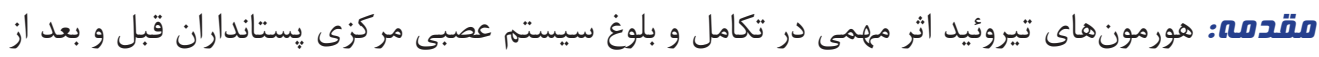

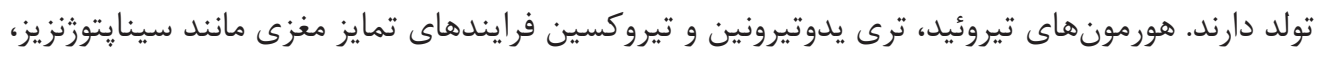

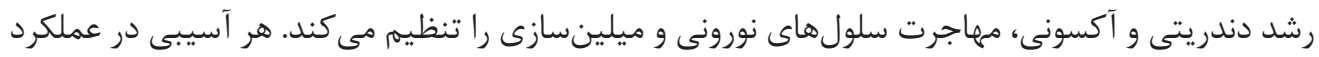

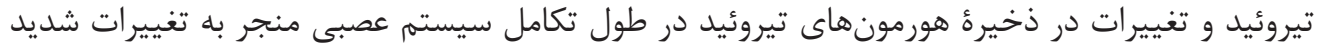

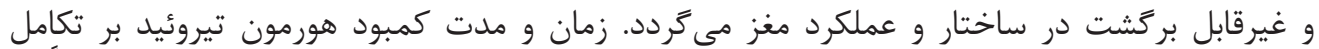

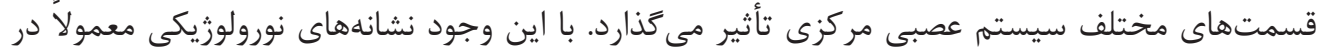

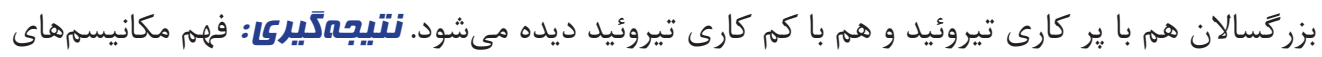

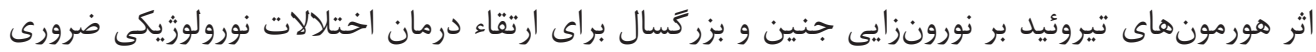
مىباشد.

ا. ا. هورمونهاى تيروئيد

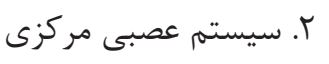

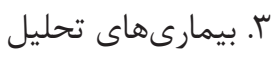

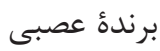

" نويسنده مسئول: فاطمه على : نور آدرس الكترونيكى: Fatemehalipoor11@gmail.com 


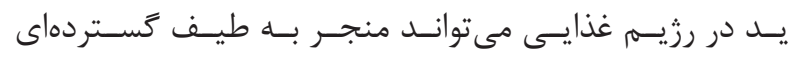
مقدمه

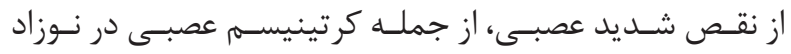

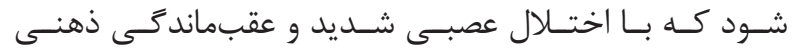

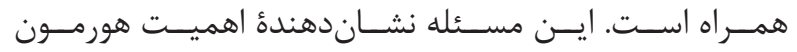

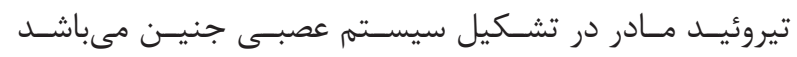

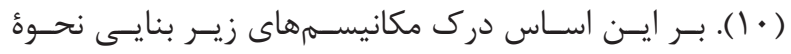

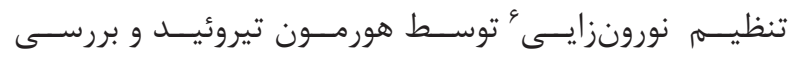

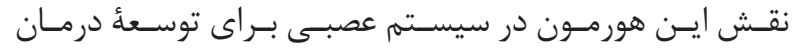

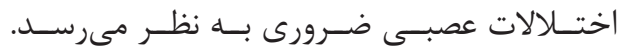

غدهُ تيروئيد

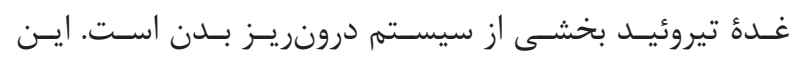

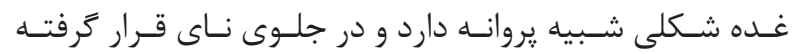

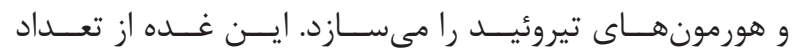

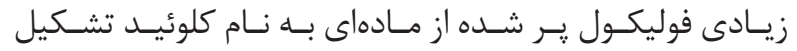

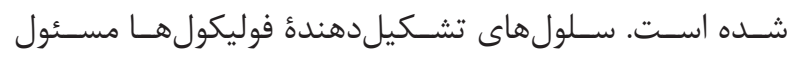

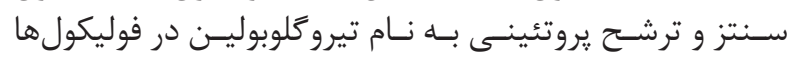

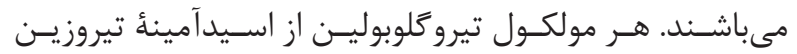

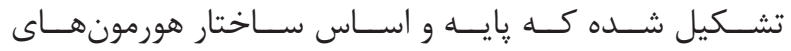

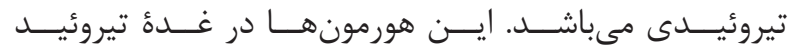

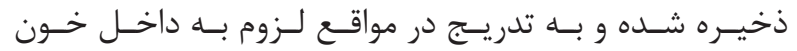

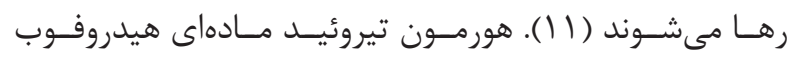

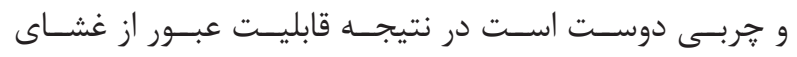

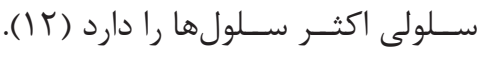

$$
\text { هورمون هاى تيروئيد }
$$

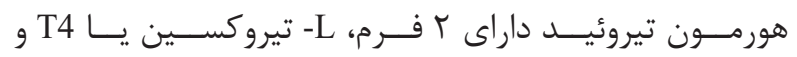

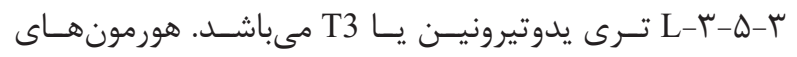

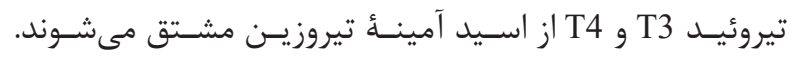

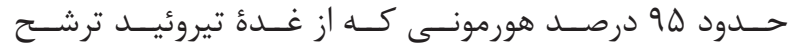

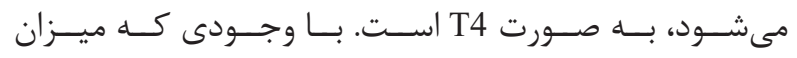

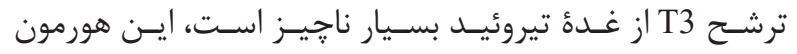

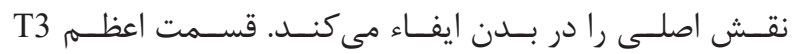

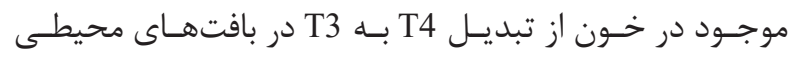

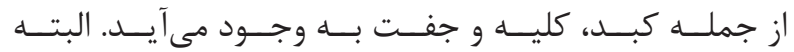

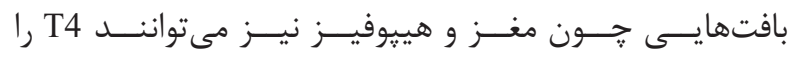

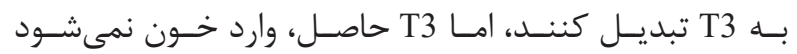

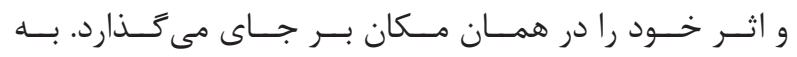

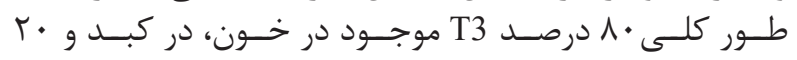

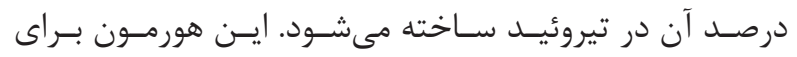

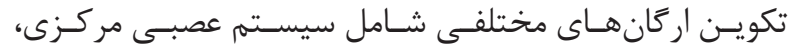

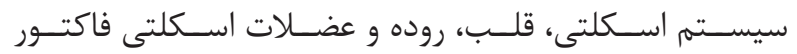

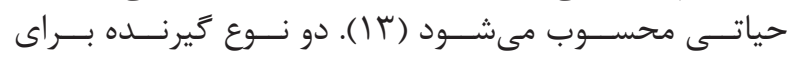

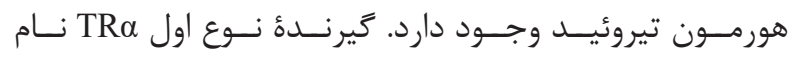

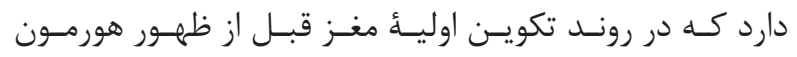

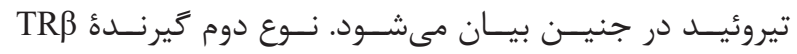

${ }^{1}$ Triiodothyronine

${ }^{2}$ Thyroxine

${ }^{3}$ Synaptogenesis

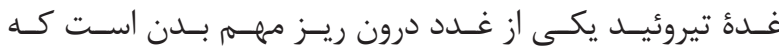

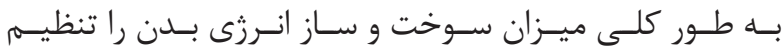

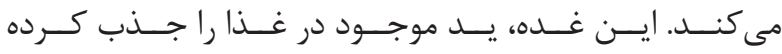

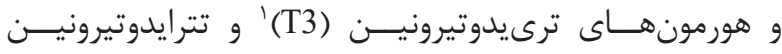

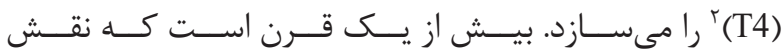

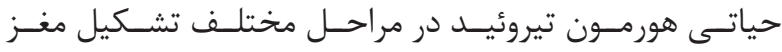

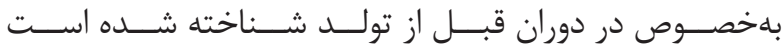

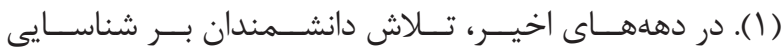

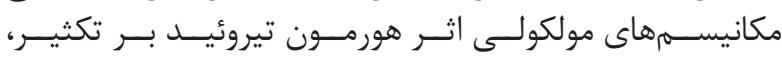

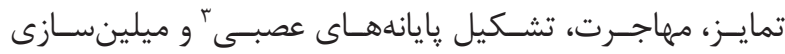

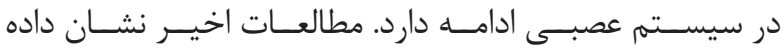

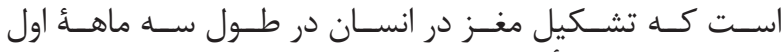

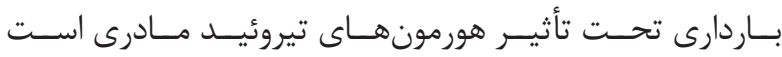

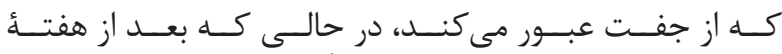

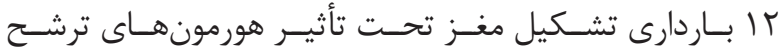

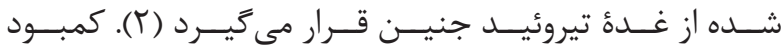

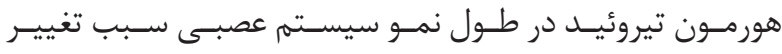

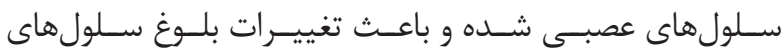

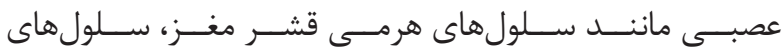

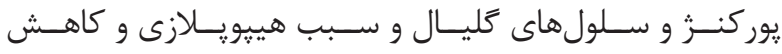

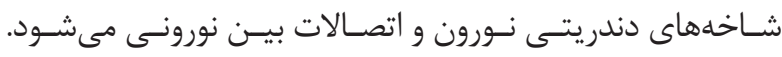

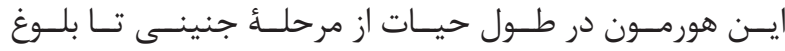

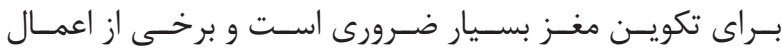

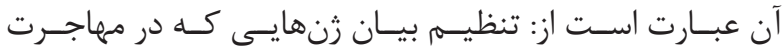

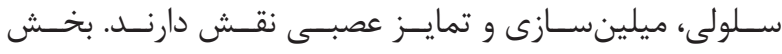

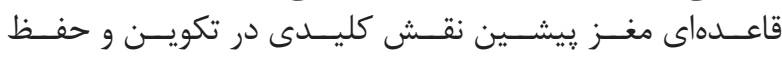

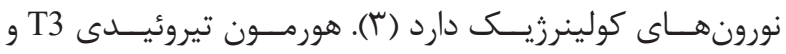

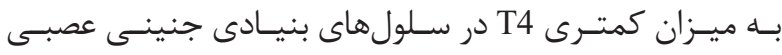

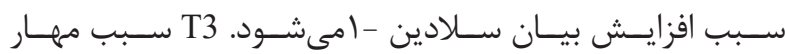

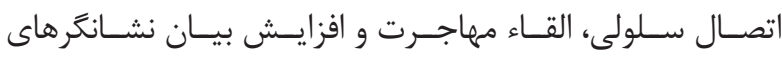

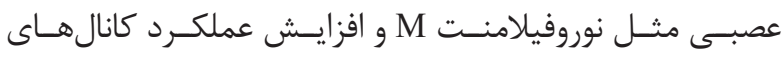

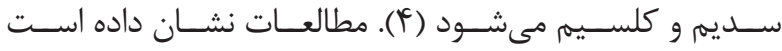

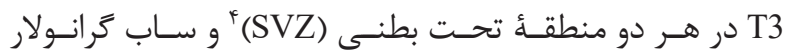

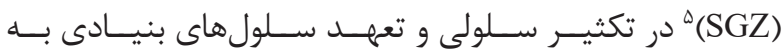

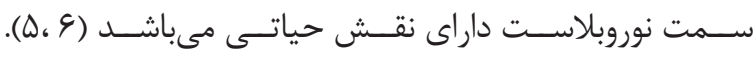

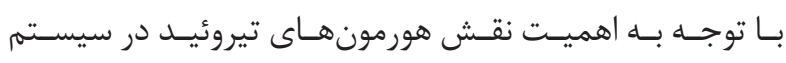

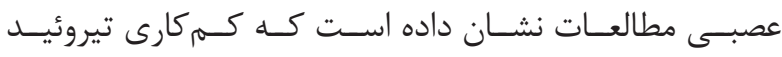

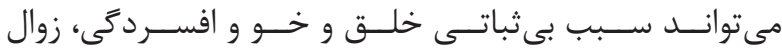

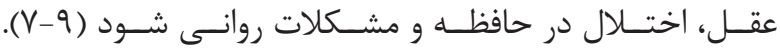

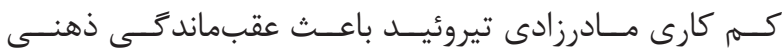

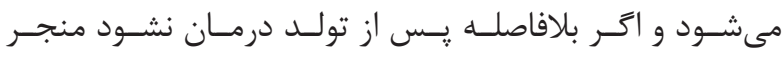

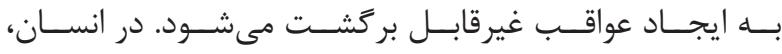

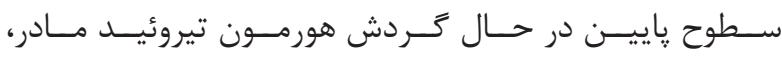

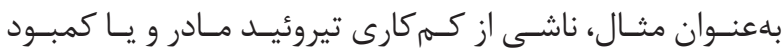

\footnotetext{
${ }^{4}$ Sub ventricular zone

${ }^{5}$ Sub granular zone

${ }^{6}$ Neurogenesis
} 


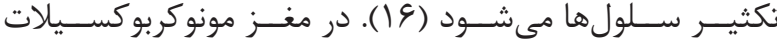

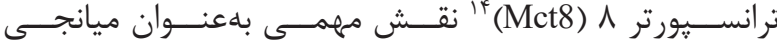

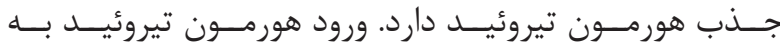

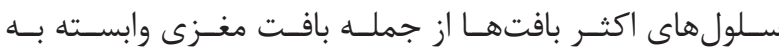

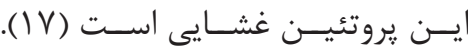

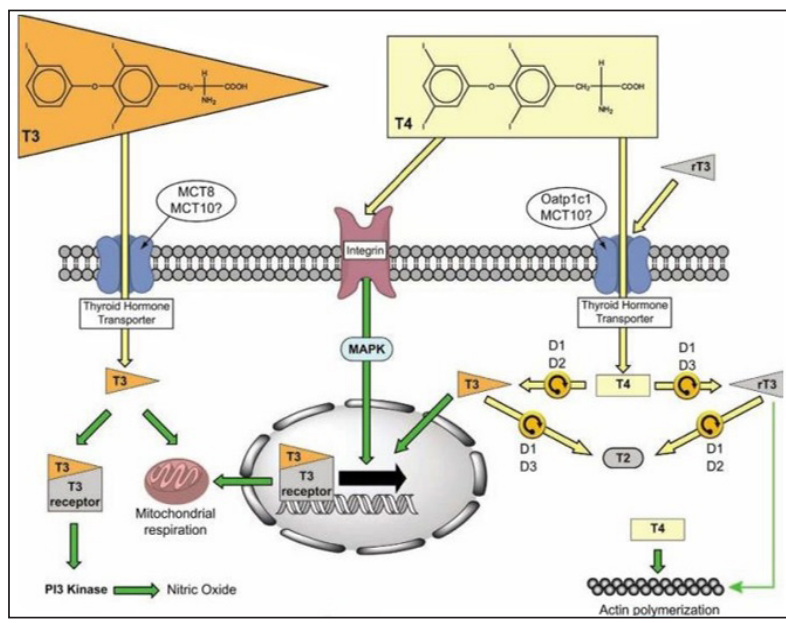

تصوير ا- عملكرد هورمون تيروئيد.

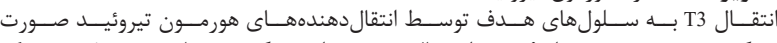

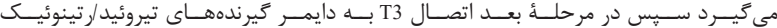

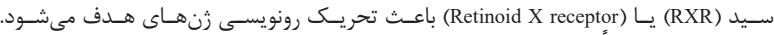

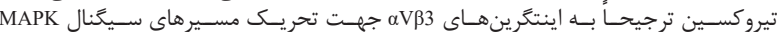

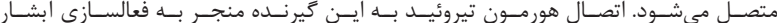

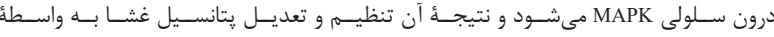

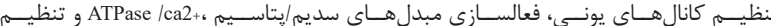
تركيبـات اسـكلت سـلولى اسـت (1) ().

$$
\text { اثر هور مون تيروئيد روى سيستم عصبى }
$$

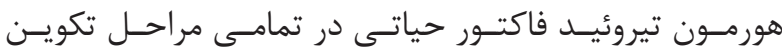

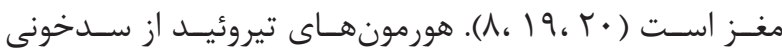

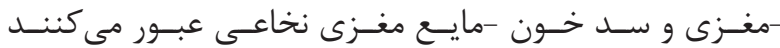

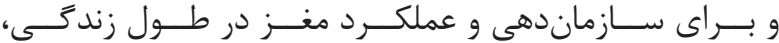

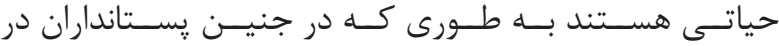

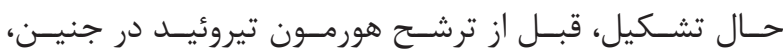

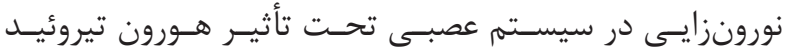

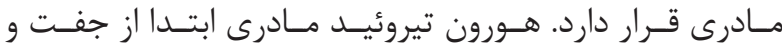

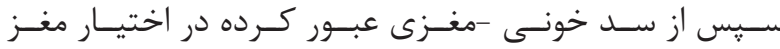

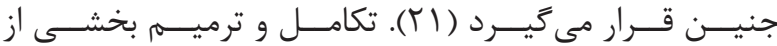

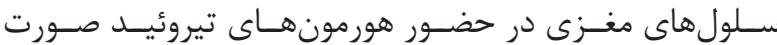

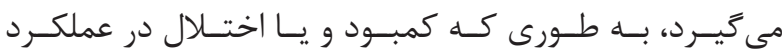

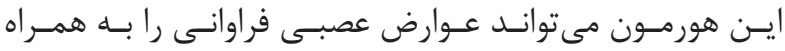

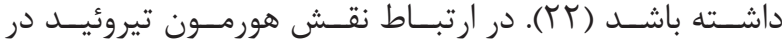

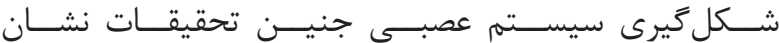

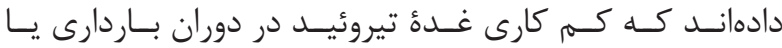

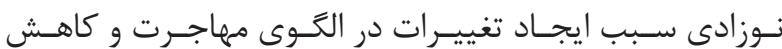

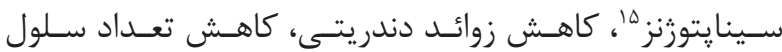

${ }^{7}$ Thyroxine-binding globulin

${ }^{8}$ Transthyretin

${ }^{9}$ Cerebrospinal fluid

${ }^{10}$ Blood brain barrier

${ }^{11}$ Iodothyronine, type II

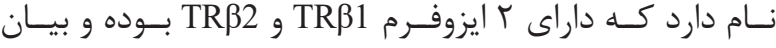

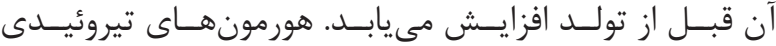

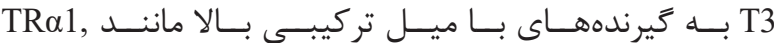

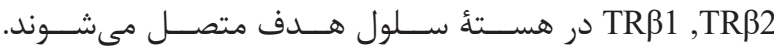

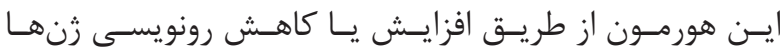

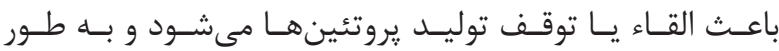

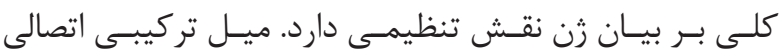

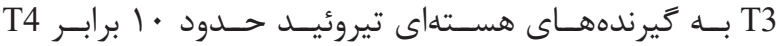

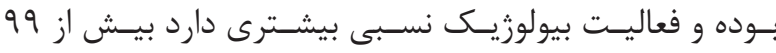

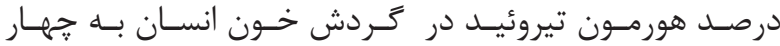

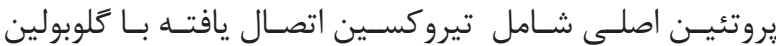

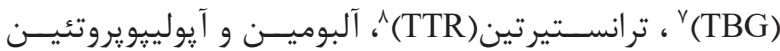

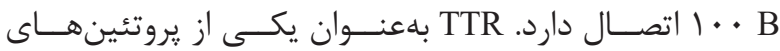

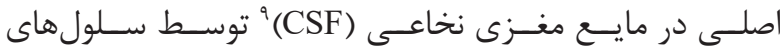

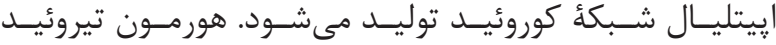

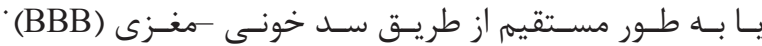

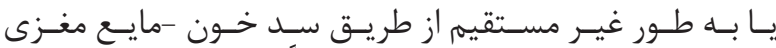

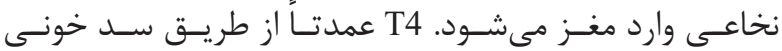

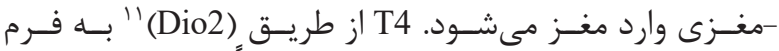

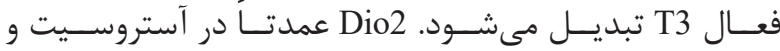

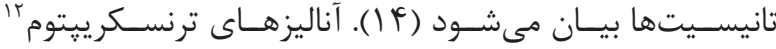

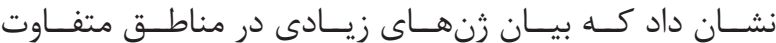

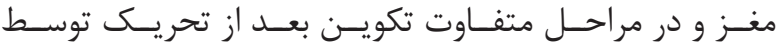

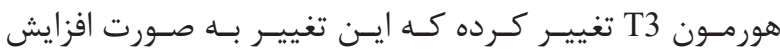

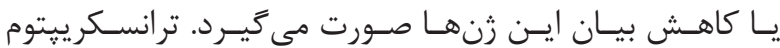

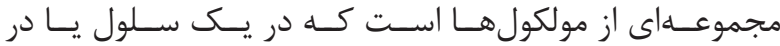

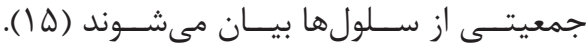
عملكرد هورمون تيروئيد در سلول

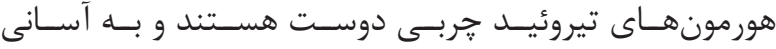

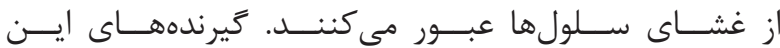

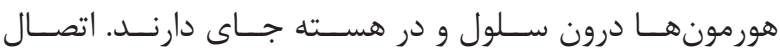

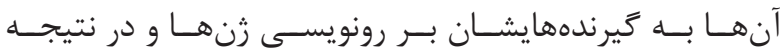

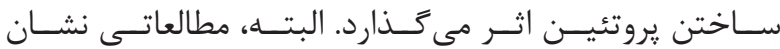

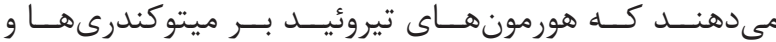

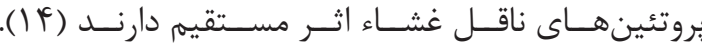

integrin $\alpha v \beta 3$

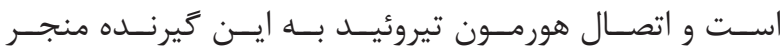

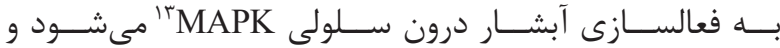

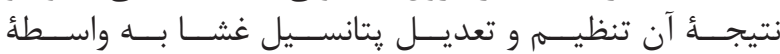

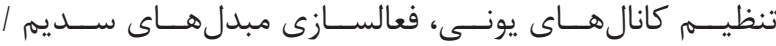

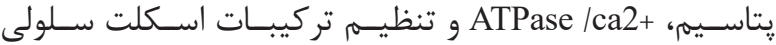

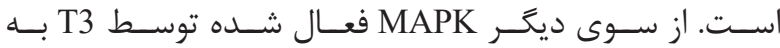

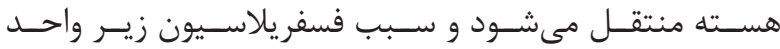

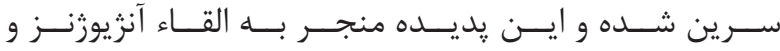

${ }^{12}$ Transcriptome

${ }^{13}$ Mitogen-activated protein kinase

${ }^{14}$ Monocarboxylate transporter 8

${ }^{15}$ Synaptogenesis 


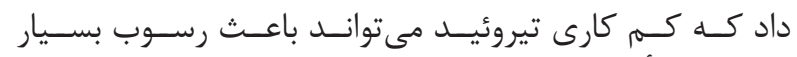

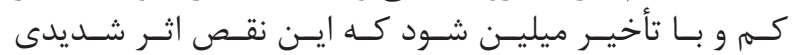

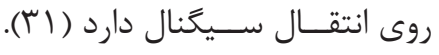

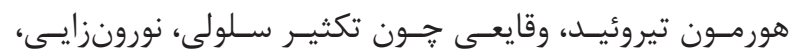

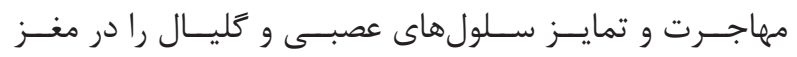

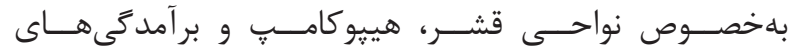

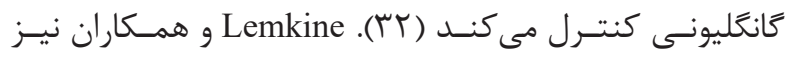

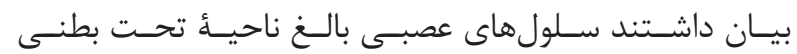

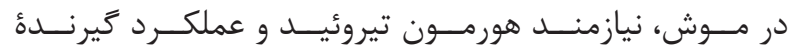

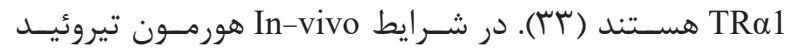

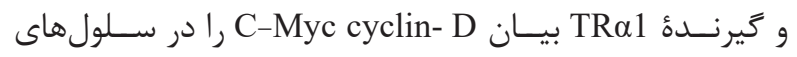

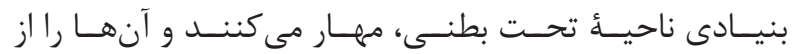

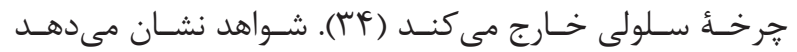

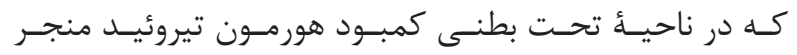

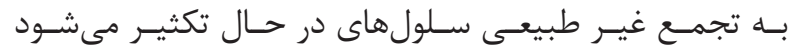

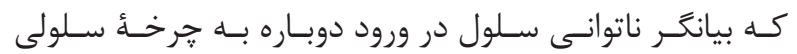

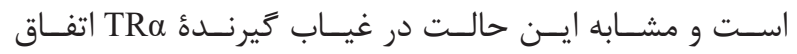

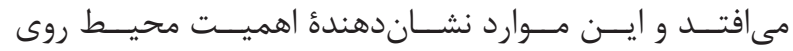

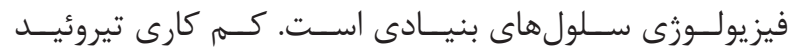

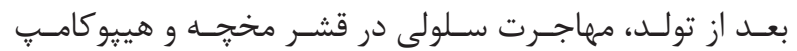

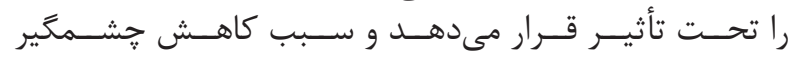

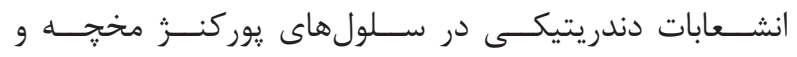

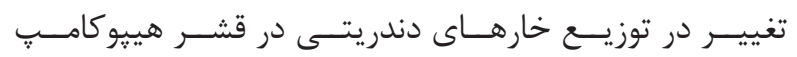

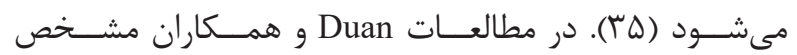

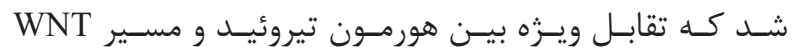

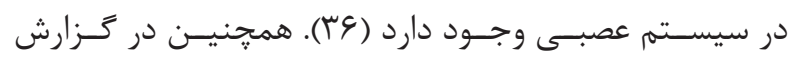

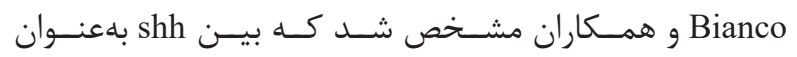

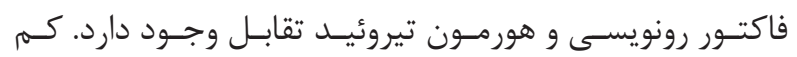

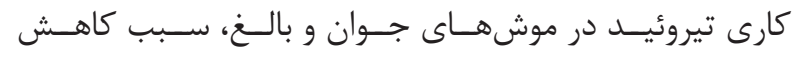

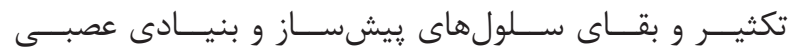

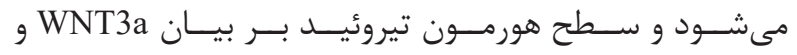

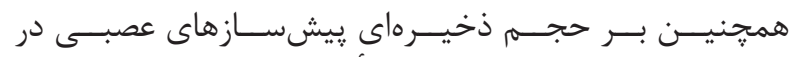

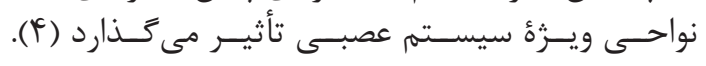

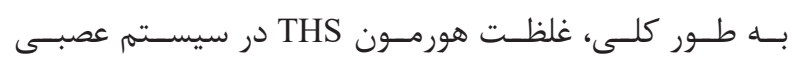

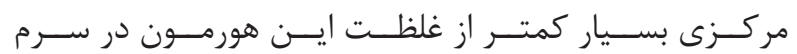

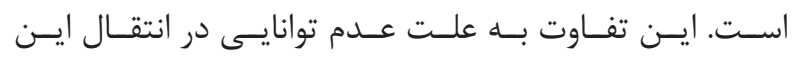

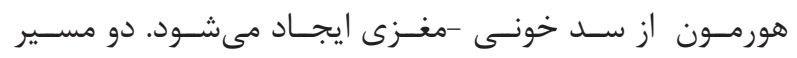

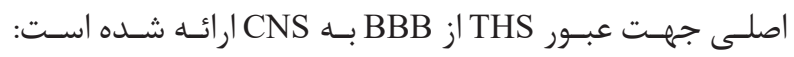

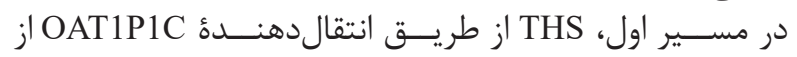

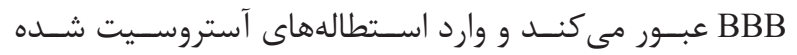

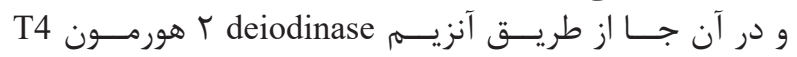

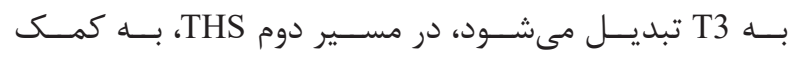

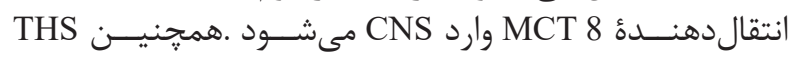

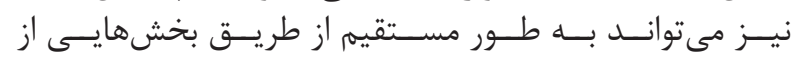

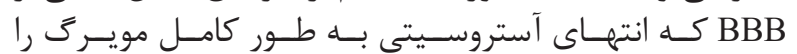

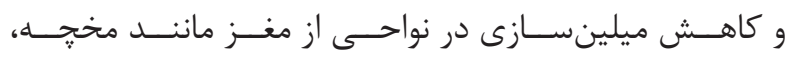

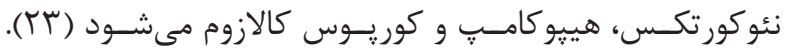

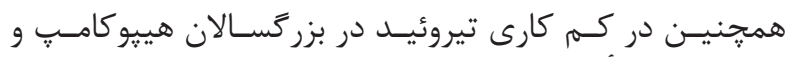

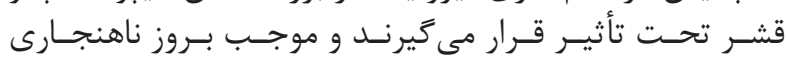

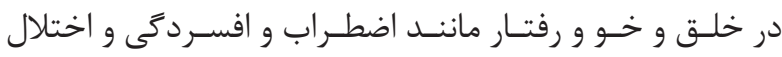

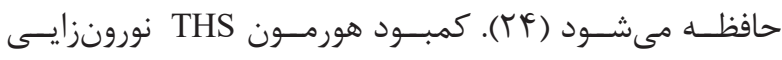

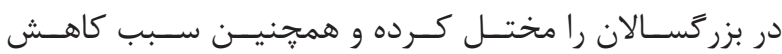

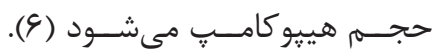

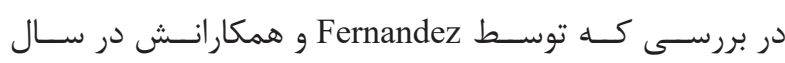

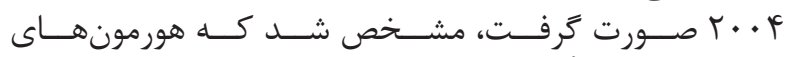

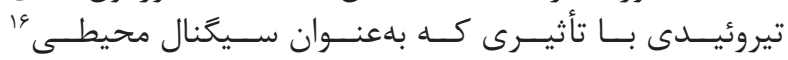

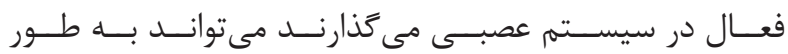

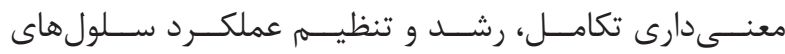

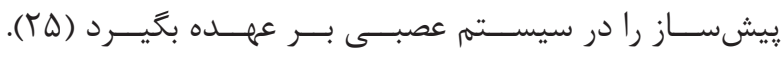

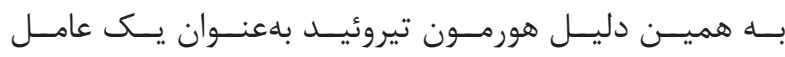

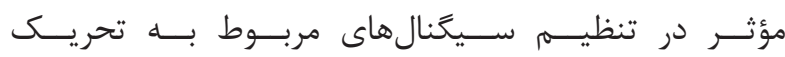

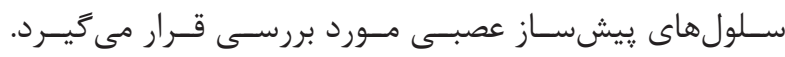

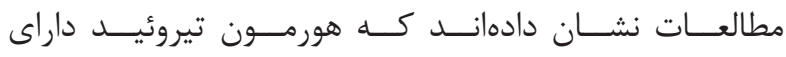

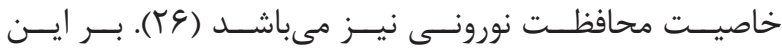

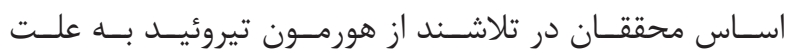

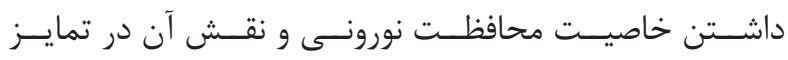

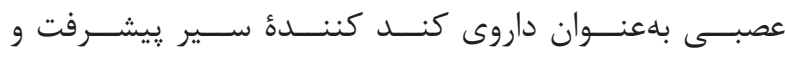

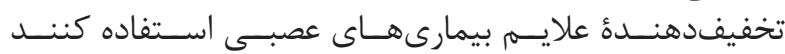

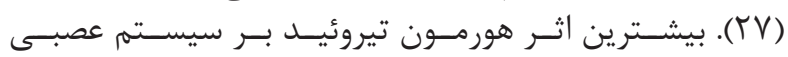

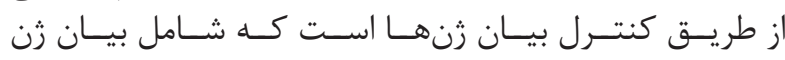

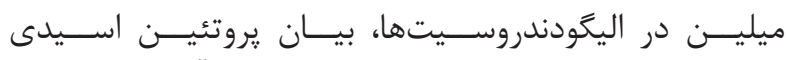

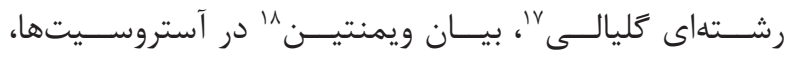

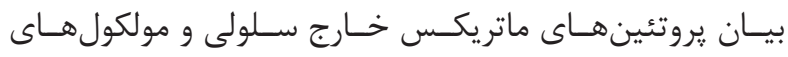

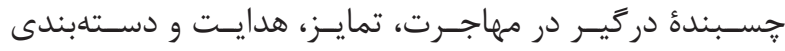

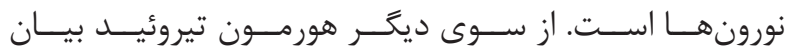

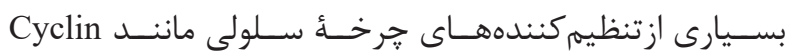

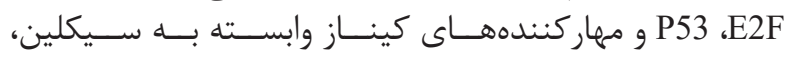

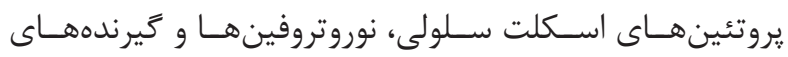

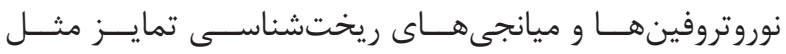

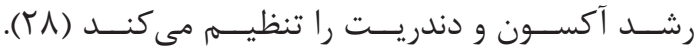

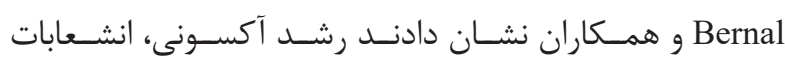

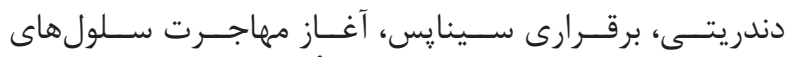

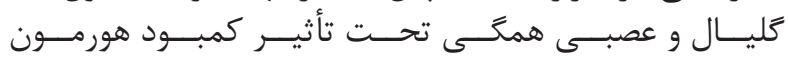

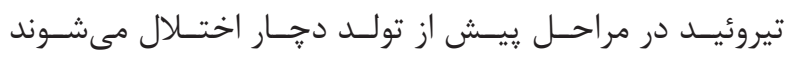

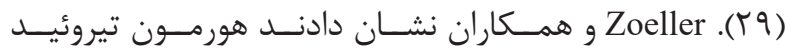

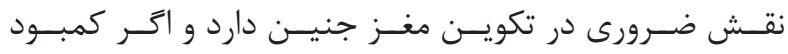

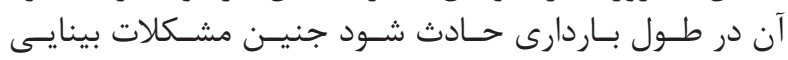

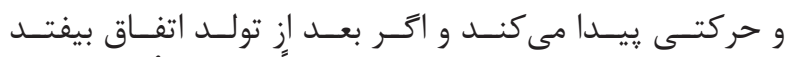

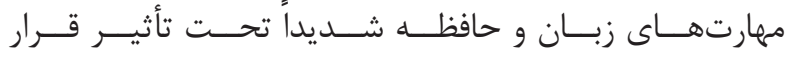

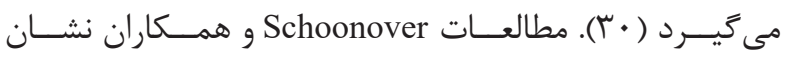


يفــاء مى كنــد. T4 نقــص در فعاليـت هورمهـون تيروئيسـد در

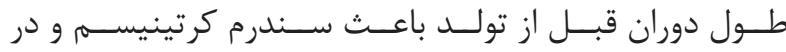

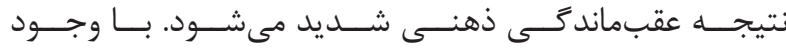

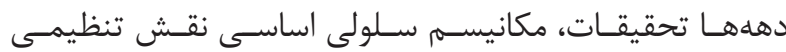

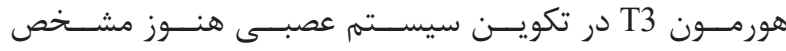

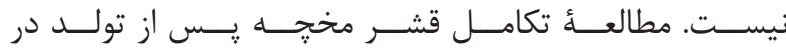

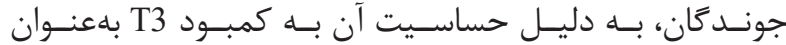

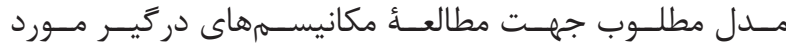

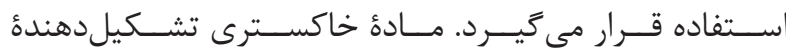

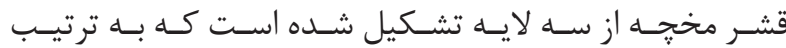

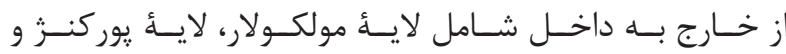

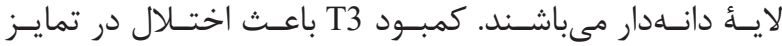

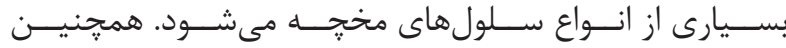

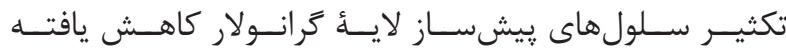

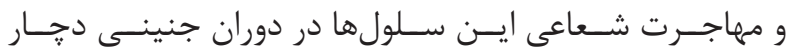

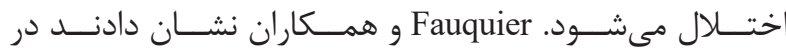

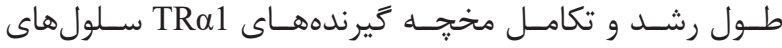

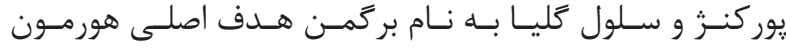

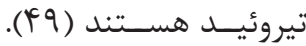

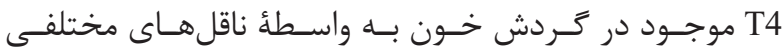

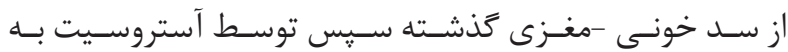

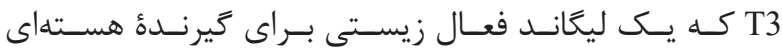

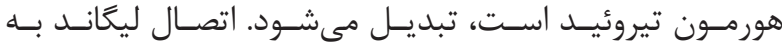

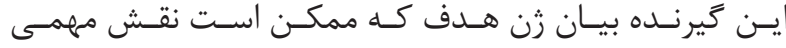

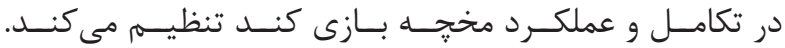

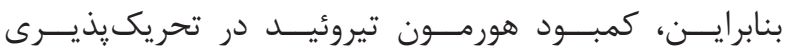

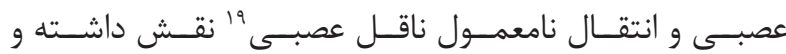

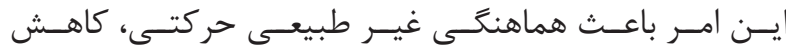

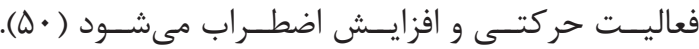

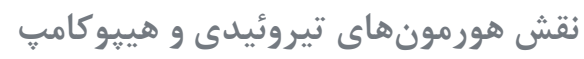

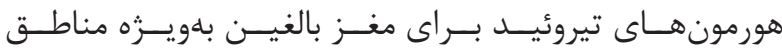

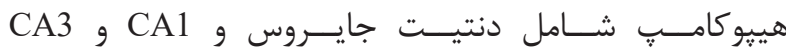

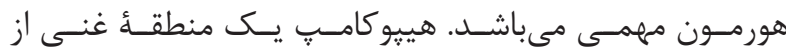

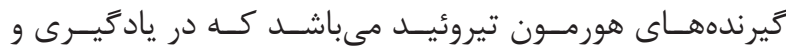

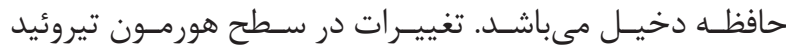

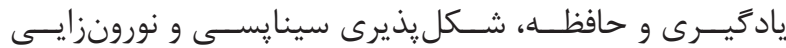

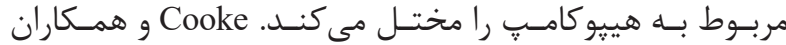

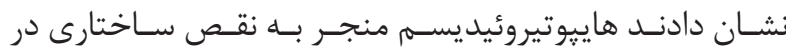

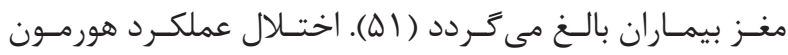

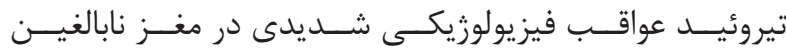

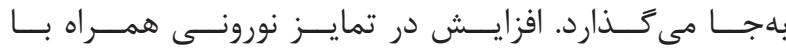

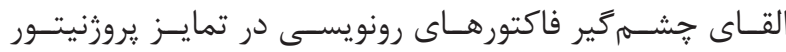

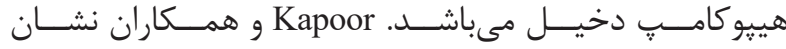

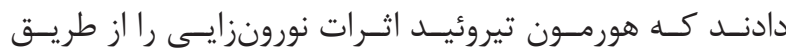

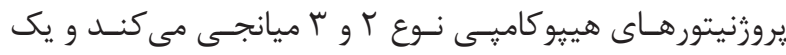

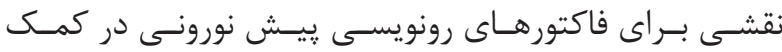

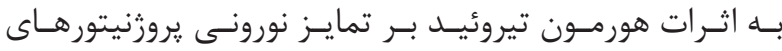

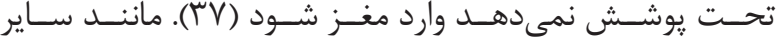

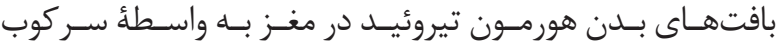

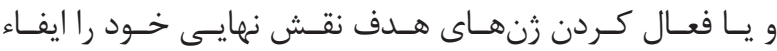

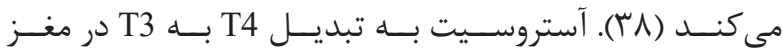

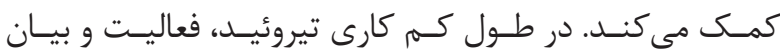

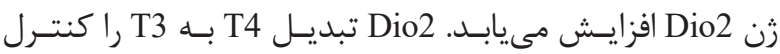

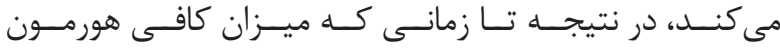

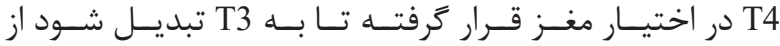

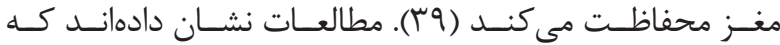

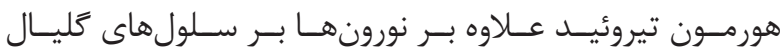

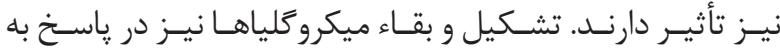

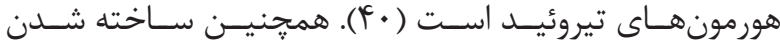

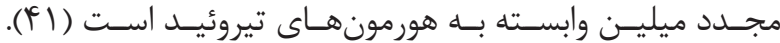

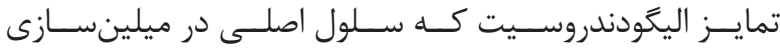

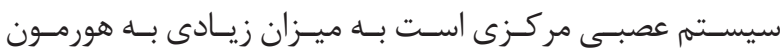

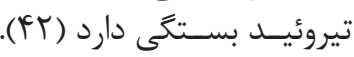

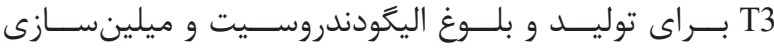

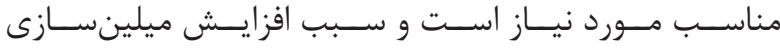

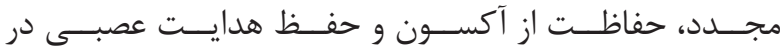

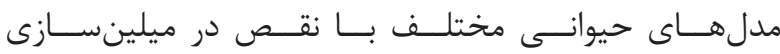

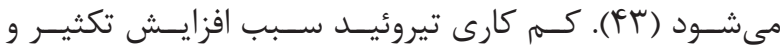

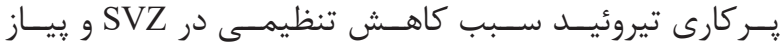

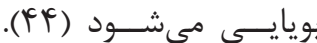

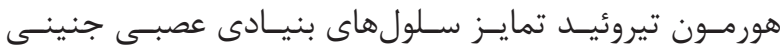

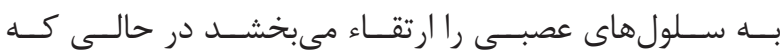

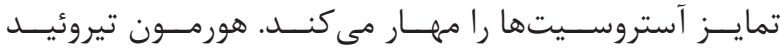

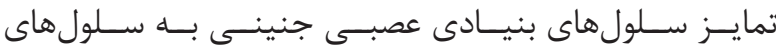

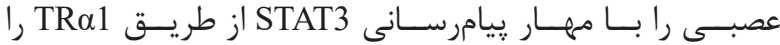

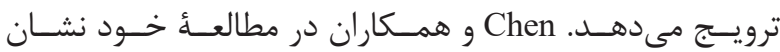

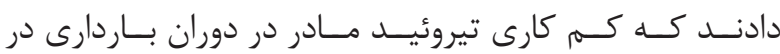

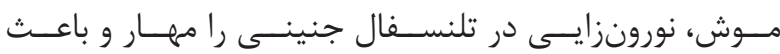

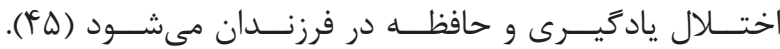

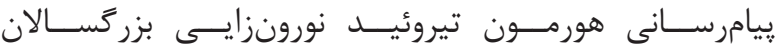

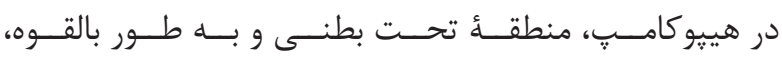

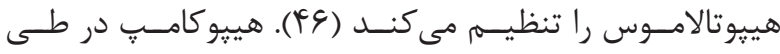

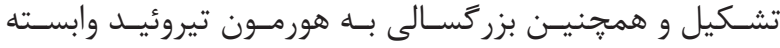

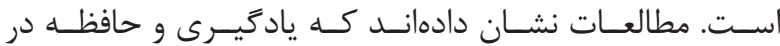

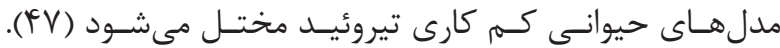

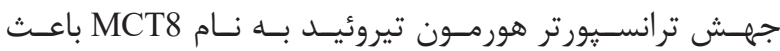

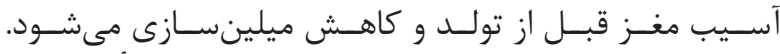

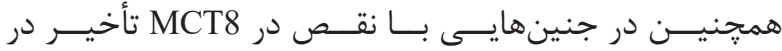

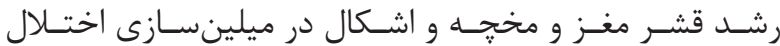

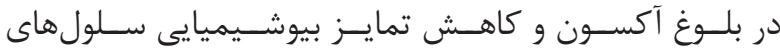

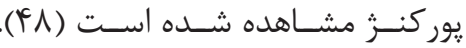
نقش هورمون تيروئيد در مخجه

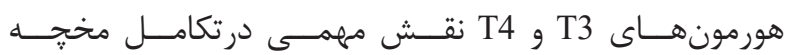




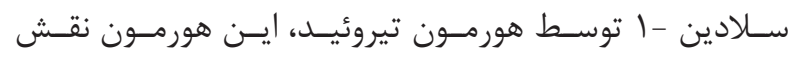

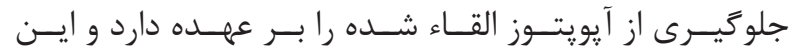

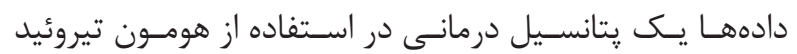

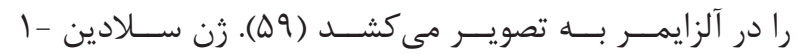

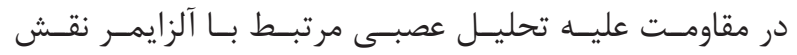

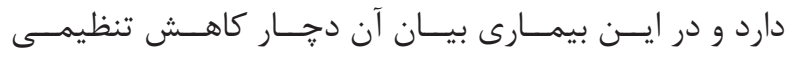

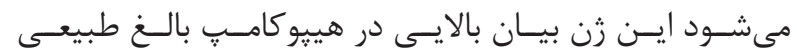

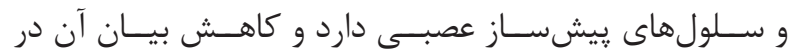

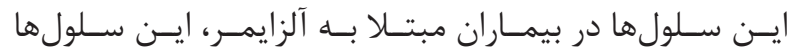

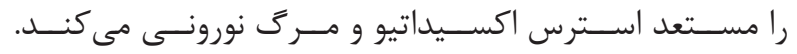
Alessandro Peri

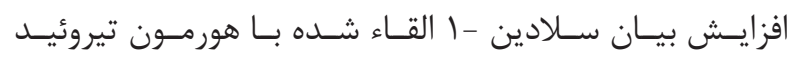

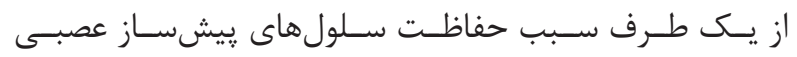

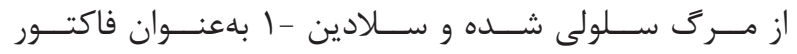

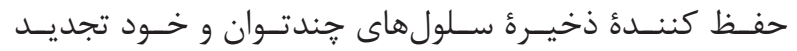

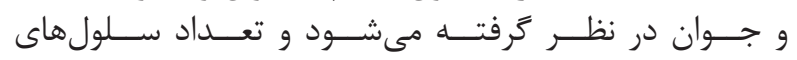

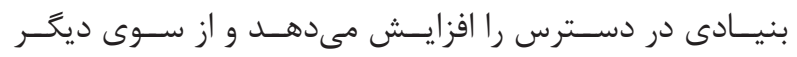

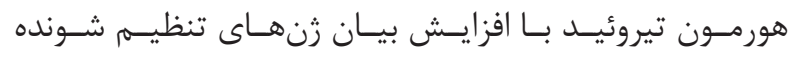

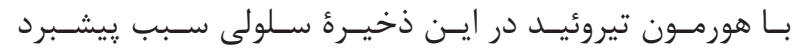

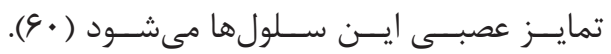

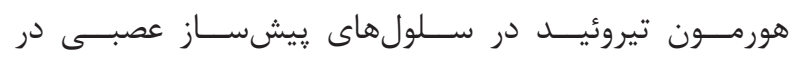

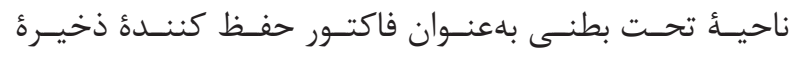

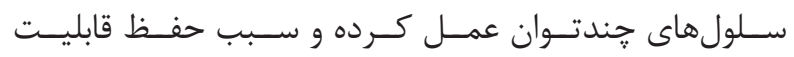

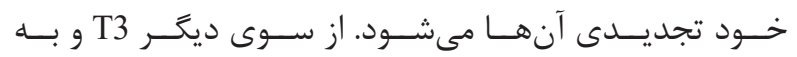

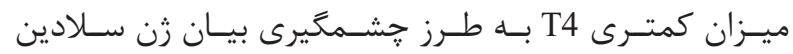

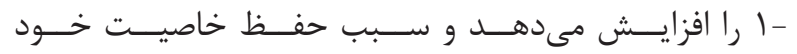

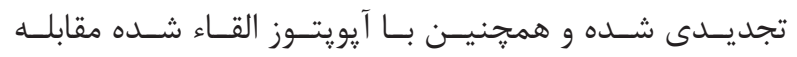

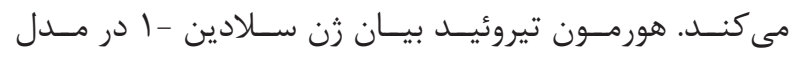

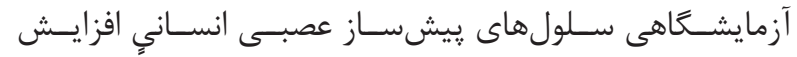

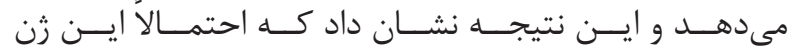

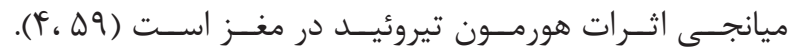

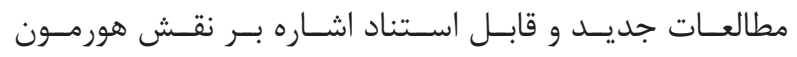

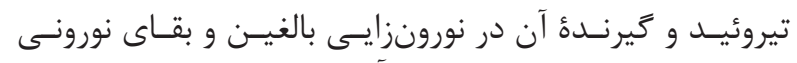

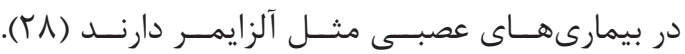

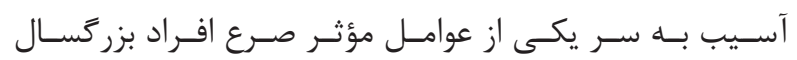

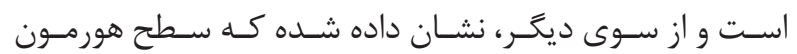

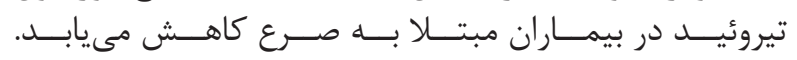

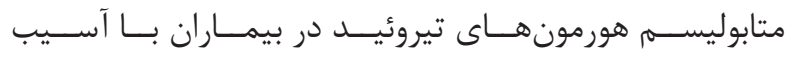

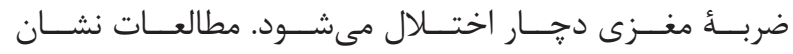

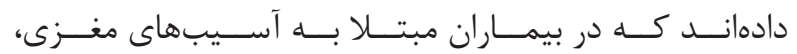

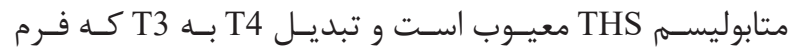

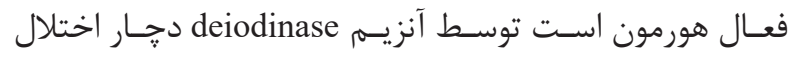

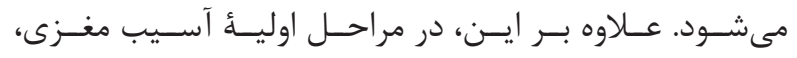

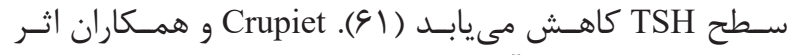

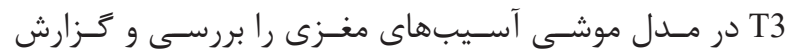

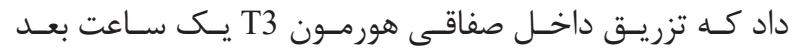

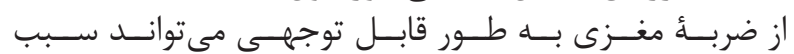

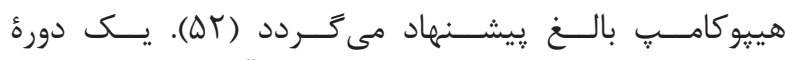

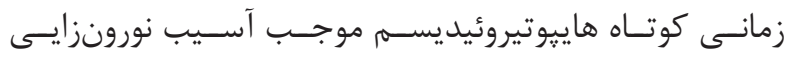

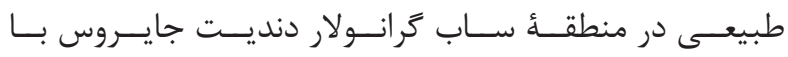

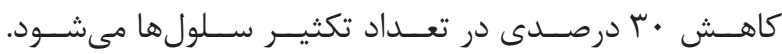

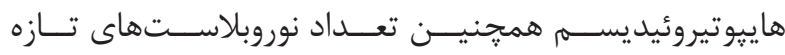

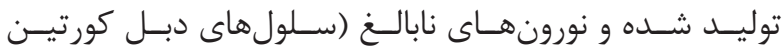

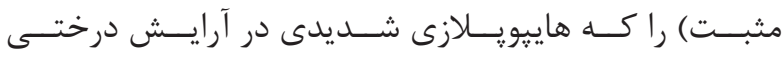

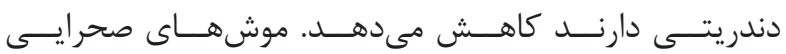

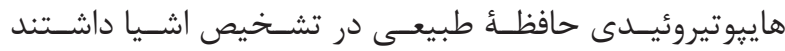

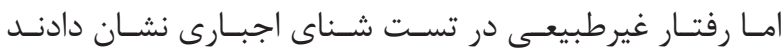

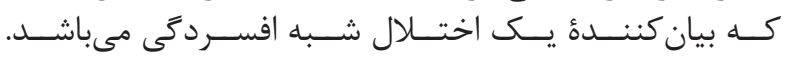

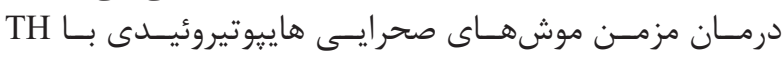

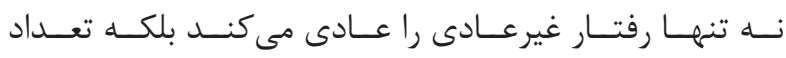

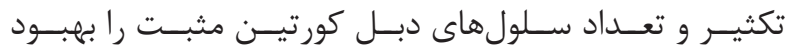

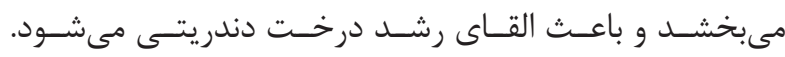

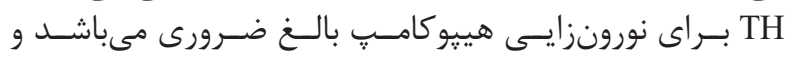

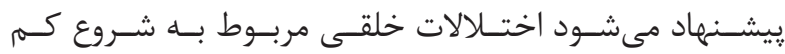

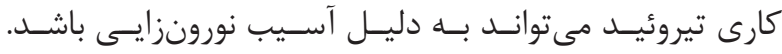

نقـــش هورمون هــــاى تيروئيـــــ در بيمارىهــــاى

عصبــى

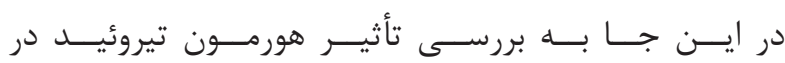

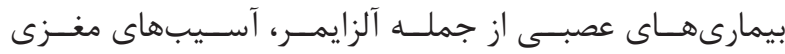

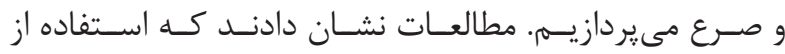

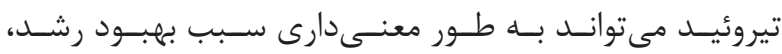

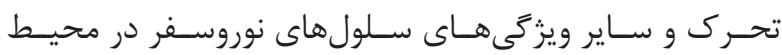

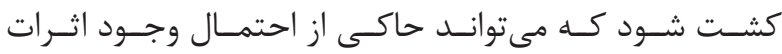

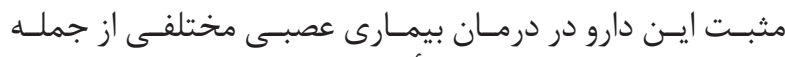

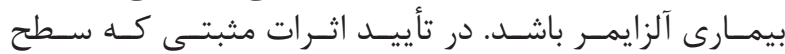

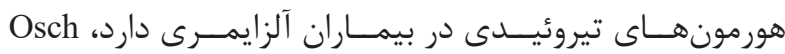

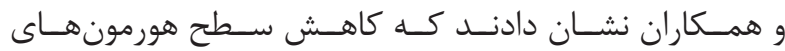

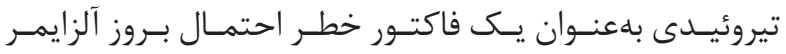

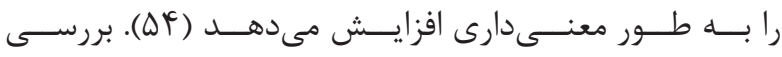

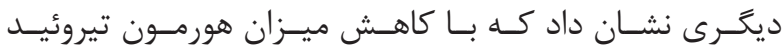

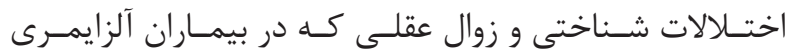

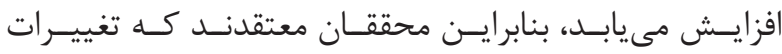

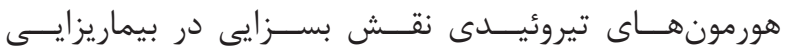

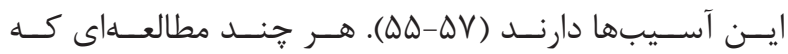

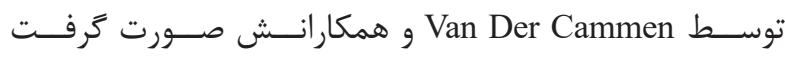

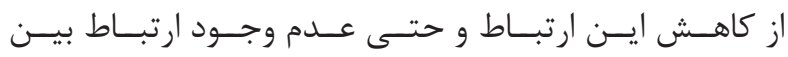

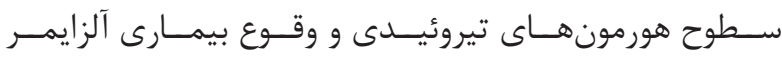

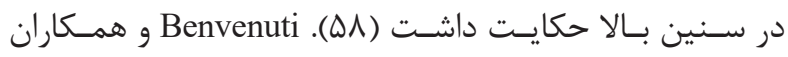

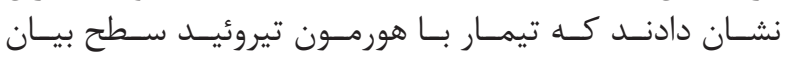

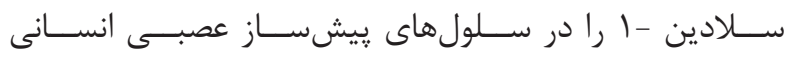

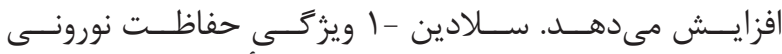

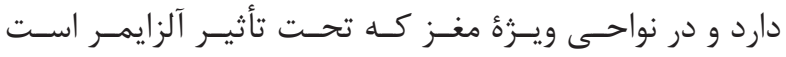

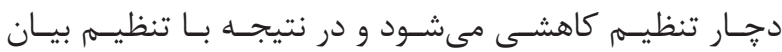




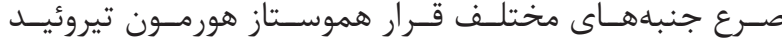

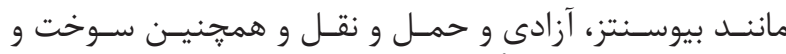

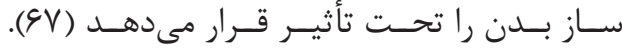

$$
\text { نتيجه كيرى }
$$

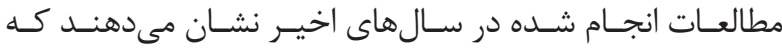

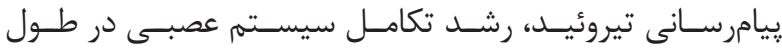

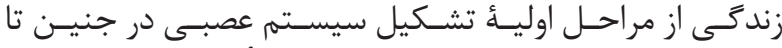

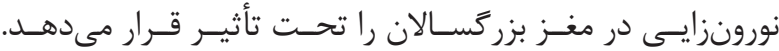

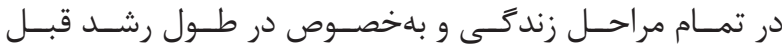

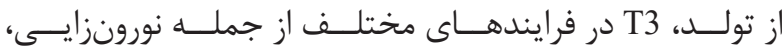

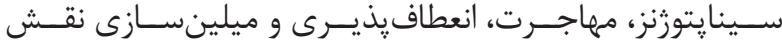

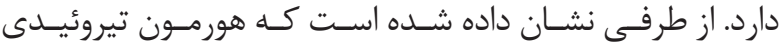

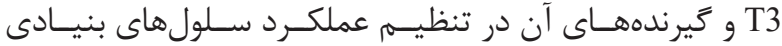

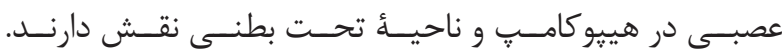

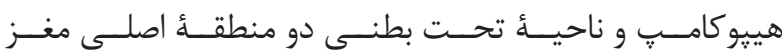

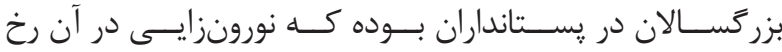

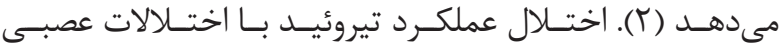

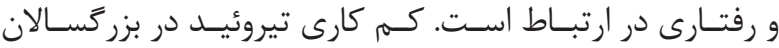

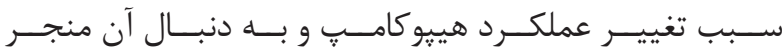

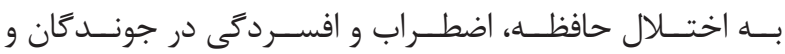

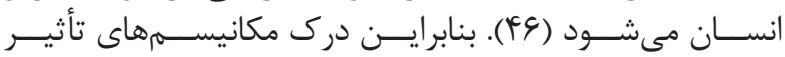

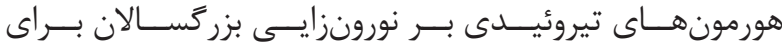

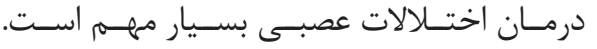

1. Mussa G, Mussa F, Bretto R, Zambelli M, Silvestro L. Influence of thyroid in nervous system growth. Minerva Pediatrica. 2001; 53(4): 325-53.

2. Cisne R, Justo R, Migowski E. Influence of thyroid hormones on nervous system development-brief review. International Journal of Basic and Applied Sciences. 2016; 5(1): 82-5.

3. Bianco AC. Metabolic effects of thyroid hormonesbeyond traditional prospects. Thyroid. 2008; 18(2): 99100.

4. Zhang L, Blomgren K, Kuhn HG, Cooper-Kuhn CM. Effects of postnatal thyroid hormone deficiency on neurogenesis in the juvenile and adult rat. Neurobiol Dis. 2009; 34(2): 366-74.

5. López-Juárez A, Remaud S, Hassani Z, Jolivet P, Simons JP, Sontag T, et al. Thyroid hormone signaling acts as a neurogenic switch by repressing Sox 2 in the adult neural stem cell niche. Cell stem cell. 2012; 10(5): 531-43.

6. Montero-Pedrazuela A, Venero C, Lavado-Autric

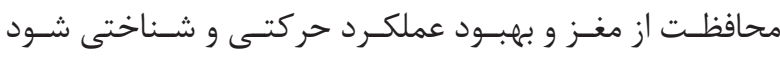

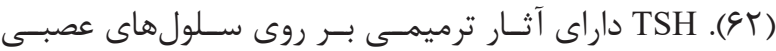

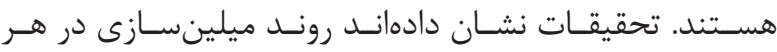

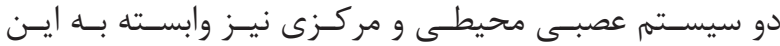

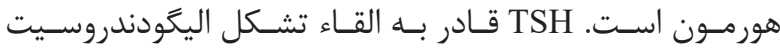

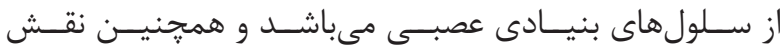

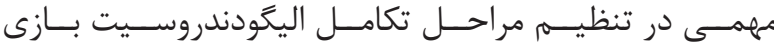

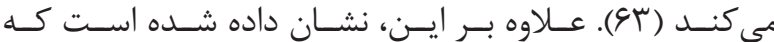

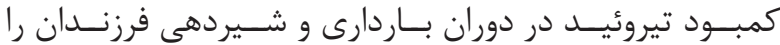

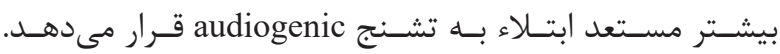

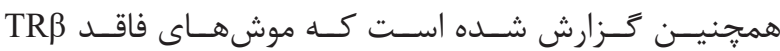

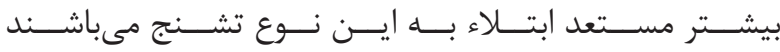
بئن (SF)

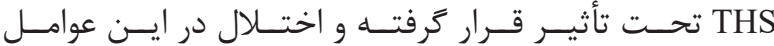

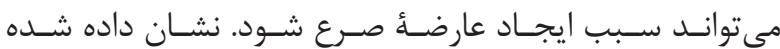

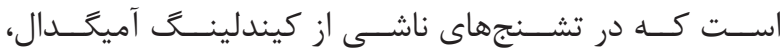

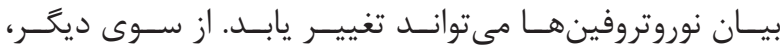

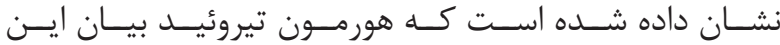

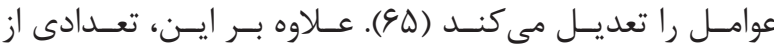

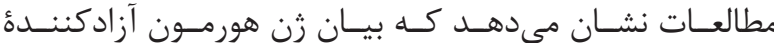

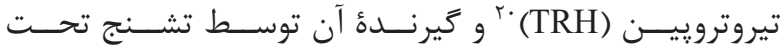

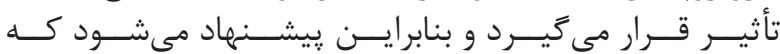
مى TRH

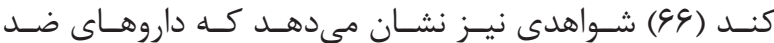

منابع

R, Fernández-Lamo I, Garcia-Verdugo J, Bernal J, et al. Modulation of adult hippocampal neurogenesis by thyroid hormones: implications in depressive-like behavior. Molecular Psychiatry. 2006; 11(4): 361-71.

7. Leach PT, Gould TJ. Thyroid hormone signaling: Contribution to neural function, cognition, and relationship to nicotine. Neurosci Biobehav Rev. 2015; 57: 252-63.

8. Ahmed OM, El-Gareib A, El-Bakry A, El-Tawab SA, Ahmed R. Thyroid hormones states and brain development interactions. Int J Dev Neurosci. 2008; 26(2): 147-209.

9. Pasand Mojdeh H, Alipour F, Borhani Haghighi M. Alzheimer's disease: background, current and future aspects. Shefaye Khatam. 2016; 4(3): 70-80

10. Stenzel D, Huttner WB. Role of maternal thyroid hormones in the developing neocortex and during human evolution. Front Neuroanat. 2013; 7(19): doi: 10.3389/ fnana.2013.00019.

11. Van Herle A, Uller R, Matthews N, Brown J. 
Radioimmunoassay for measurement of thyroglobulin in human serum. J Clin Invest. 1973; 52(6): 1320-7.

12. Verhoeven AJ, Kamer P, Groen AK, Tager JM. Effects of thyroid hormone on mitochondrial oxidative phosphorylation. Biochem J. 1985; 226(1): 183-92.

13. Kandel ER, Schwartz JH, Jessell TM. Principles of neural science: McGraw-Hill New York; 2000.

14. Wirth EK, Schweizer U, Kohrle J. Transport of thyroid hormone in brain. Front Endocrinol (Lausanne). 2014; 5: 98. doi: 10.3389/fendo.2014.00098.

15. Takahashi T, Gasch A, Nishizawa N, Chua N-H. The DIMINUTO gene of Arabidopsis is involved in regulating cell elongation. Genes Dev. 1995; 9(1): 97107.

16. Davis PJ, Goglia F, Leonard JL. Nongenomic actions of thyroid hormone. Nat Rev Endocrinol. 2016; 12(2): 111-21.

17. Visser WE, Mullem AA, Visser TJ, Peeters RP. Different causes of reduced sensitivity to thyroid hormone: diagnosis and clinical management. Clin Endocrinol (Oxf). 2013; 79(5): 595-605.

18. Kansagra SM, McCudden CR, Willis MS. The challenges and complexities of thyroid hormone replacement. Laboratory Medicine. 2010; 41(6): 338-48.

19. Begum N, Sussman KE, Draznin B. Differential effects of diabetes on adipocyte and liver phosphotyrosine and phosphoserine phosphatase activities. Diabetes. 1991; 40(12): 1620-9.

20. Zoeller R, Rovet J. Timing of thyroid hormone action in the developing brain: clinical observations and experimental findings. J Neuroendocrinol. 2004; 16(10): 809-18.

21. de Escobar GM, Obregón MJ, del Rey FE. Iodine deficiency and brain development in the first half of pregnancy. Public Health Nutrition. 2007; 10(12A): 1554-70.

22. Smith JW, Evans AT, Costall B, Smythe JW. Thyroid hormones, brain function and cognition: a brief review. Neurosci Biobehav Rev. 2002; 26(1): 45-60.

23. Gilbert ME, Rovet J, Chen Z, Koibuchi N. Developmental thyroid hormone disruption: prevalence, environmental contaminants and neurodevelopmental consequences. Neurotoxicology. 2012; 33(4): 842-52.

24. Bernal J, Guadaño-Ferraz A, Morte B. Thyroid hormone transporters [mdash] functions and clinical implications. Nature Reviews Endocrinology. 2015; 11(7): 406-17.

25. Fernandez M, Pirondi S, Manservigi M, Giardino L, Calzà L. Thyroid hormone participates in the regulation of neural stem cells and oligodendrocyte precursor cells in the central nervous system of adult rat. European Journal of Neuroscience. 2004; 20(8): 2059-70.

26. Bunevicius A, Iervasi G, Bunevicius R. Neuroprotective actions of thyroid hormones and low-T3 syndrome as a biomarker in acute cerebrovascular disorders. Expert Review of Neurotherapeutics. 2015; 15(3): 315-26.

27. Tan ZS, Vasan RS. Thyroid function and Alzheimer's disease. J Alzheimers Dis. 2009; 16(3): 503-7.

28. Klahre U, Noguchi T, Fujioka S, Takatsuto S, Yokota $\mathrm{T}$, Nomura $\mathrm{T}$, et al. The arabidopsis DIMINUTO/ DWARF1 gene encodes a protein involved in steroid synthesis. The Plant Cell. 1998; 10(10): 1677-90.

29. Hoyer S, Müller D, Plaschke K. Desensitization of brain insulin receptor effect on glucose/energy and related metabolism. Springer; 1994.

30. Laudet V, Hänni C, Coll J, Catzeflis F, Stéhelin D. Evolution of the nuclear receptor gene superfamily. The EMBO Journal. 1992; 11(3): 1003.

31. Bianco AC, Kim BW. Deiodinases: implications of the local control of thyroid hormone action. Journal of Clinical Investigation. 2006; 116(10): 2571.

32. Cuevas E, Ausó E, Telefont M, De Escobar GM, Sotelo C, Berbel P. Transient maternal hypothyroxinemia at onset of corticogenesis alters tangential migration of medial ganglionic eminence-derived neurons. European Journal of Neuroscience. 2005; 22(3): 541-51.

33. Lemkine G, Raj A, Alfama G, Turque N, Hassani Z, Alegria-Prevot $\mathrm{O}$, et al. Adult neural stem cell cycling in vivo requires thyroid hormone and its alpha receptor. The FASEB Journal. 2005; 19(7): 863-5.

34. Clevers $H$. Wnt/ $\beta$-catenin signaling in development and disease. Cell. 2006; 127(3): 469-80.

35. Bernal J. Thyroid hormone receptors in brain development and function. Nature Clinical Practice Endocrinology \& Metabolism. 2007; 3(3): 249-59.

36. Duan X, Kang E, Liu CY, Ming G-1, Song H. Development of neural stem cell in the adult brain. Current Opinion in Neurobiology. 2008; 18(1): 108-15.

37. Schroeder AC, Privalsky ML. Thyroid hormones, t3 


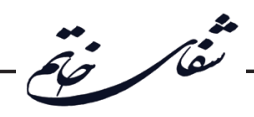

and 4 , in the brain. Frontiers in Endocrinology. 2013; 5: 40

38. Brent GA. Mechanisms of thyroid hormone action. The Journal of Clinical Investigation. 2012; 122(9): 3035-43.

39. Roman C, Fuior EV, Trusca VG, Kardassis D, Simionescu M, Gafencu AV. Thyroid hormones upregulate apolipoprotein E gene expression in astrocytes. Biochemical and Biophysical Research Communications. 2015; 468(1): 190-5.

40. Schweizer U, Köhrle J. Function of thyroid hormone transporters in the central nervous system. Biochimica et Biophysica Acta (BBA)-General Subjects. 2013; 1830(7): 3965-73.

41. Calzà L, Fernandez M, Giardino L. Cellular approaches to central nervous system remyelination stimulation: thyroid hormone to promote myelin repair via endogenous stem and precursor cells. Journal of Molecular Endocrinology. 2010; 44(1): 13-23.

42. Hung P-L, Huang C-C, Huang H-M, Tu D-G, Chang Y-C. Thyroxin treatment protects against white matter injury in the immature brain via brain-derived neurotrophic factor. Stroke. 2013; 44(8): 2275-83.

43. Dell'Acqua M, Lorenzini L, D’Intino G, Sivilia S, Pasqualetti P, Panetta V, et al. Functional and molecular evidence of myelin-and neuroprotection by thyroid hormone administration in experimental allergic encephalomyelitis. Neuropathology and Applied Neurobiology. 2012; 38(5): 454-70.

44. Fernandez M, Pirondi S, Manservigi M, Giardino L, Calza L. Thyroid hormone participates in the regulation of neural stem cells and oligodendrocyte precursor cells in the central nervous system of adult rat. Eur $\mathrm{J}$ Neurosci. 2004; 20(8): 2059-70.

45. Chen C, Zhou Z, Zhong M, Zhang Y, Li M, Zhang L, et al. Thyroid hormone promotes neuronal differentiation of embryonic neural stem cells by inhibiting STAT3 signaling through TR $\alpha 1$. Stem Cells and Development. 2012; 21(14): 2667-81.

46. Remaud S, Gothié J-D, Morvan-Dubois G, Demeneix BA. Thyroid hormone signaling and adult neurogenesis in mammals. Front Endocrinol. 2014; 5(62): 10.3389.

47. Willoughby KA, McAndrews MP, Rovet JF. Effects of maternal hypothyroidism on off spring hippocampus and memory. Thyroid. 2014; 24(3): 576-84.

48. López-Espíndola D, Morales-Bastos C, Grijota-
Martínez C, Liao X-H, Lev D, Sugo E, et al. Mutations of the thyroid hormone transporter MCT8 cause prenatal brain damage and persistent hypomyelination. The Journal of Clinical Endocrinology \& Metabolism. 2014; 99(12): E2799-E804

49. Fauquier T, Chatonnet F, Picou F, Richard S, Fossat $\mathrm{N}$, Aguilera N, et al. Purkinje cells and Bergmann glia are primary targets of the $\mathrm{TR} \alpha 1$ thyroid hormone receptor during mouse cerebellum postnatal development. Development. 2014; 141(1): 166-75.

50. Koibuchi N. The role of thyroid hormone on functional organization in the cerebellum. The Cerebellum. 2013; 12(3): 304-6.

51. Cooke GE, Mullally S, Correia N, O’Mara SM, Gibney J. Hippocampal volume is decreased in adults with hypothyroidism. Thyroid. 2014; 24(3): 433-40.

52. Kapoor R, Desouza LA, Nanavaty IN, Kernie SG, Vaidya VA. Thyroid hormone accelerates the differentiation of adult hippocampal progenitors. J Neuroendocrinol. 2012; 24(9): 1259-71.

53. Montero-Pedrazuela A, Venero C, Lavado-Autric R, Fernandez-Lamo I, Garcia-Verdugo JM, Bernal J, et al. Modulation of adult hippocampal neurogenesis by thyroid hormones: implications in depressive-like behavior. Mol Psychiatry. 2006; 11(4): 361-71.

54. van Osch LA, Hogervorst E, Combrinck M, Smith AD. Low thyroid-stimulating hormone as an independent risk factor for Alzheimer disease. Neurology. 2004; 62(11): 1967-71.

55. Davis JD, Podolanczuk A, Donahue JE, Stopa E, Hennessey JV, Luo L-G, et al. Thyroid hormone levels in the prefrontal cortex of post-mortem brains of Alzheimer's disease patients. Current Aging Science. 2008; 1(3): 175-81.

56. Tan ZS, Vasan RS. Thyroid function and Alzheimer's disease. Journal of Alzheimer's Disease: JAD. 2008; 16(3): $503-7$.

57. Ewins DL, Rossor MN, Butler J, Rogues PK, Mullen MJ, McGregor AM. Association between autoimmune thyroid disease and familial Alzheimers disease. Clinical Endocrinology. 1991; 35(1): 93-6.

58. Van Der Cammen TJ, Raso FM, Van Harskamp F, De Jager MC. Lack of association between thyroid disorders

and alzheimer's disease in older persons: a crosssectional observational study in a geriatric outpatient population. Journal of the American Geriatrics Society. 2003; 51(6): 884. 
59. Benvenuti S, Luciani P, Cellai I, Deledda C, Baglioni $\mathrm{S}$, Saccardi R, et al. Thyroid hormones promote cell differentiation and up-regulate the expression of the seladin-1 gene in vitro models of human neuronal precursors. Journal of Endocrinology. 2008; 197(2): 437-46.

60. Peri A, Serio M. Neuroprotective effects of the Alzheimer's disease-related gene seladin-1. Journal of Molecular Endocrinology. 2008; 41(5): 251-61.

61. Fernandez-Rodriguez E, Bernabeu I, Castro AI, Casanueva FF. Hypopituitarism after traumatic brain injury. Eur J Endocrinol. 2015; 44(1): 151-9.

62. Crupi R, Paterniti I, Campolo M, Di Paola R, Cuzzocrea S, Esposito E. Exogenous T3 administration provides neuroprotection in a murine model of traumatic brain injury. Pharmacol Res. 2013; 70(1): 80-9.

63. Fernandez M, Paradisi M, Del Vecchio G, Giardino L, Calza L. Thyroid hormone induces glial lineage of primary neurospheres derived from non-pathological and pathological rat brain: implications for remyelinationenhancing therapies. Int J Dev Neurosci. 2009; 27(8): 769-78.

64. Jankowsky JL, Patterson PH. The role of cytokines and growth factors in seizures and their sequelae. Progress in Neurobiology. 2001; 63(2): 125-49.

65. Kim S-Y, Smith MA, Post RM, Rosen JB. Attenuation of kindling-induced decreases in NT-3 mRNA by thyroid hormone depletion. Epilepsy Res. 1998; 29(3): 211-20

66. Kubek M, Knoblach S, Sharif N, Burt D, Buterbaugh G, Fuson K. Thyrotropin-releasing hormone gene expression and receptors are differentially modified in limbic foci by seizures. Ann Neurol. 1993; 33(1): 70-6.

67. Svalheim S, Sveberg L, Mochol M, Taubøll E. Interactions between antiepileptic drugs and hormones. Seizure. 2015; 28: 12-7. 